\title{
ADAPTAÇÃO DE PRÓTESES AUDITIVAS NO CANDIDATO AO IMPLANTE COCLEAR
}

\section{Fitting hearing aid in patients candidate for cochlear implants}

\author{
Ana Tereza de Matos Magalhães ${ }^{(1)}$, Maria Valéria Goffi-Gomez ${ }^{(2)}$, Isabela Jardim ${ }^{(3)}$, \\ Robinson Koji Tsuji (4), Rubens de Brito Neto ${ }^{(5)}$, Ricardo Ferreira Bento ${ }^{(6)}$
}

\section{RESUMO}

Objetivo: caracterizar o perfil audiométrico e demográfico de pacientes candidatos ao implante coclear encaminhados ao setor de prótese auditiva e entre esses, a frequência de indivíduos que se beneficiaram da amplificação sonora. Métodos: foram estudados os pacientes atendidos no período de maio de 2007 a dezembro 2008. Foi realizado o levantamento do perfil da população segundo sexo, idade, escolaridade e etiologia. Foi calculada a média da melhor e pior orelha e classificada segundo Frota (2003). A média dos limiares auditivos com as próteses auditivas indicadas foi calculada e considerada como benefício quando permitia acesso aos sons da fala do português brasileiro (Russo e Behlau, 1993). Resultados: foram avaliados nesse período 194 prontuários. 108 pacientes já eram usuários de próteses auditivas (55,6\%), 100 do sexo masculino (52\%), 94 do sexo feminino (48\%), 109 eram crianças (56\%) e 85 eram adultos (44\%). A média de idade foi 4,8 anos (crianças) e 41,9 anos (adultos). Entre os adultos, 24 possuíam ensino fundamental incompleto (33\%). A etiologia mais frequente foi a desconhecida (33\%). Dezenove pacientes se beneficiaram do uso da prótese auditiva (10\%). No grupo de pacientes que se beneficiaram da prótese auditiva, a média dos limiares em campo livre com amplificação foi de 47 dBNA e 48 dBNA, na melhor e na pior orelha, respectivamente, nas crianças e 50 dBNA e 45 dBNA, respectivamente, nos adultos. Conclusão: o perfil dos pacientes foi: na maioria criança, sexo masculino, etiologia desconhecida, já usuários de próteses auditivas com média de idade 4,8 anos (crianças) e 41,9 anos (adultos), audiometria com perda auditiva neurossensorial profunda bilateral, sendo que a prótese beneficiou $10 \%$ dos pacientes.

DESCRITORES: Implante Coclear; Audição; Auxiliares de Audição; Perda Auditiva; Fala

(1) Fonoaudióloga da Equipe de Implante Coclear do Hospital das Clínicas da Faculdade de Medicina da Universidade de São Paulo, HC-FMUSP, São Paulo, SP, Brasil; Mestranda pela Faculdade de Medicina da Universidade de São Paulo.

(2) Fonoaudióloga; Hospital das Clínicas da Faculdade de Medicina da Universidade de São Paulo, HC-FMUSP, São Paulo, SP, Brasil; Doutora em Distúrbios da Comunicação Humana (Fonoaudiologia) pela Universidade Federal de São Paulo.

(3) Fonoaudióloga; Fundação Otorrinolaringologia da Faculdade de Medicina da Universidade de São Paulo, FMUSP, São Paulo, SP, Brasil; Doutora em Ciências da Saúde pela Universidade de São Paulo.

(4) Médico Otorrinolaringologista; Hospital das Clínicas da Faculdade de Medicina da Universidade de São Paulo, HC-FMUSP, São Paulo, SP, Brasil; Doutor em Ciências da Saúde pela Universidade de São Paulo.

(5) Médico Otorrinolaringologista; Hospital das Clínicas da Faculdade de Medicina da Universidade de São Paulo, HCFMUSP, São Paulo, SP, Brasil; Livre Docência pelo Hospital das Clínicas da Faculdade de Medicina da Universidade de São Paulo.

\section{INTRODUÇÃO}

A perda auditiva severa a profunda bilateral é uma deficiência que foi considerada durante muito tempo como alteração em que o individuo era incapaz de comunicar-se ou até mesmo ouvir sons ambientais como alarmes e sirenes, tornando-o socialmente incapacitante. Atualmente, os sistemas de amplificação sonora vêm sendo desenvolvidos e a tecnologia constantemente aprimorada na tentativa de melhorar a qualidade de vida destes indivíduos integrando-os na sociedade ${ }^{1}$.

(6) Médico Otorrinolaringologista; Hospital das Clínicas da Faculdade de Medicina da Universidade de São Paulo, HC-FMUSP, São Paulo, SP, Brasil; Livre Docência pela Universidade de São Paulo.

Conflito de interesses: inexistente 
O uso do implante coclear é considerado o tratamento ouro para indivíduos com perda severa a profunda bilateral ${ }^{2}$ de diferentes faixas etárias, desde crianças pequenas até adultos ${ }^{3}$.

Para a indicação do implante coclear, torna-se necessária uma avaliação multidisciplinar minuciosa incluindo avaliação médica e fonoaudiológica composta por uma bateria de exames clínicos, audiológicos completos além de testes de percepção de fala com prótese auditiva adequada e adaptada bilateralmente ${ }^{4}$.

No programa de implante coclear do Hospital das Clínicas da Faculdade de Medicina da Universidade de São Paulo (HC-FMUSP) pelo Sistema Único de Saúde o processo de avaliação e seleção dos candidatos ao implante coclear tem como objetivos: verificar a experiência auditiva com algum tipo de amplificação sonora e caso seja usuário de prótese auditiva, averiguar o desempenho e benefício que possui, além de verificar o comprometimento do paciente ou do familiar com o acompanhamento do uso da prótese auditiva ${ }^{4,5}$.

A etapa de protetização é fundamental para verificar se o candidato possui critérios audiológicos para o implante coclear. Além disso, atualmente existe uma tendência para implantar indivíduos com resíduos auditivos. O estudo de Gordon et al. (2001) ${ }^{6}$ mostra que os melhores resultados são em pacientes com maiores resíduos, motrando a importância de uma boa adaptação pré e pós-cirúrgica. Após a cirurgia, o indivíduo poderá usar a prótese auditiva contralateral ao implante coclear.

Os benefícios da estimulação bimodal, isto é, implante coclear combinado com uso de prótese auditiva no lado contralateral, são descritos em vários estudos, como exemplo, percepção de fala no ruído, localização sonora e nas situações de comunicação diária ${ }^{7-10}$.

$\mathrm{Na}$ prática clínica, na primeira avaliação, observa-se que grande parte destes indivíduos tem seu processo de avaliação interrompido necessitando de atendimento no setor de prótese auditiva. Dentre os motivos estão: não possuírem experiência sonora monoaural ou binaural e em algum momento de suas vidas adquirirem experiência negativa ou estarem com as próteses auditivas com defeito, má conservação ou inadequadas para o uso.

O trabalho teve como objetivo traçar o perfil audiométrico e demográfico dos pacientes candidatos ao implante coclear encaminhados ao setor de prótese auditiva, entre esses, a frequência de indivíduos que se beneficiaram da amplificação sonora.

\section{MÉTODOS}

O estudo foi observacional e retrospectivo, sendo realizado por meio de prontuários de pacientes atendidos pelo setor de prótese auditiva encaminhados pela equipe de implante coclear do HCFMUSP no período de maio de 2007 a dezembro de 2008.

Para o estudo foram considerados os seguintes critérios de inclusão:

- indivíduos cadastrados e aceitos para avaliação no programa de implante coclear do HC-FMUSP;

- perda auditiva neurossensorial severa a profunda bilateral, segundo Frota (2003) ${ }^{11}$;

- pacientes encaminhados para o setor de prótese auditiva no programa de implante coclear;

- seguimento completo do acompanhamento.

Para identificar o fluxo de pacientes convocados encaminhados para o setor de prótese auditiva no período de maio de 2007 a dezembro de 2008, foram levantados todos os pacientes cadastrados e atendidos no programa neste período e destes pacientes, quantos foram encaminhados para o setor de prótese auditiva.

Foram coletados dados dos prontuários sobre idade, sexo, escolaridade, etiologia, audiometria tonal, limiares auditivos em campo livre, histórico do uso de prótese auditiva e conduta da Equipe de Implante Coclear. Foram consideradas crianças as pessoas com idade de 0 a 17 anos e 11 meses e adultos, aqueles maiores que 18 anos; a escolaridade foi separada por: ensino fundamental (completo e incompleto), ensino médio (completo e incompleto), ensino superior (completo e incompleto) e pós-graduação (completo e incompleto).

No levantamento da etiologia, foram retirados dados do prontuário médico. Foram listadas apenas as etiologias recorrentes no estudo.

Os dados da audiometria tonal limiar foram retirados dos prontuários ou realizados no setor de prótese auditiva, com audiômetro Madsen Midimate 622. Foram considerados os limiares em cada frequência de 250, 500, 1000, 2000, 4000,6000 e $8000 \mathrm{~Hz}$, e agrupados por melhor e pior orelha com os seguintes cirtérios: as melhores médias nos limiares de 500, 1000, 2000 e $4000 \mathrm{~Hz}$. Quando fossem iguais, o critério de desempate seria a média de 6000 e $8000 \mathrm{~Hz}$ e quando mesmo assim fosse igual, a melhor orelha foi considerada a direita. Foram excluídos os pacientes que não possuíam estes dados e/ou não foi possível realizar a pesquisa dos limiares auditivos. Para o cálculo da média dos limiares, o valor de $130 \mathrm{~dB}$ foi considerado como ausência de resposta na intensidade máxima do audiômetro (120dB). 


\begin{tabular}{|c|c|c|c|c|c|c|}
\hline $250 \mathrm{~Hz}$ & $500 \mathrm{~Hz}$ & $1000 \mathrm{~Hz}$ & $2000 \mathrm{~Hz}$ & $4000 \mathrm{~Hz}$ & $6000 \mathrm{~Hz}$ & $8000 \mathrm{~Hz}$ \\
\hline $80 \mathrm{~dB}$ & $90 \mathrm{~dB}$ & $110 \mathrm{~dB}$ & $110 \mathrm{~dB}$ & $110 \mathrm{~dB}$ & $100 \mathrm{~dB}$ & $80 \mathrm{~dB}$ \\
\hline
\end{tabular}

Figura 1 - Limite máximo de saída do amplificador para audiometria em campo livre (em dBNA)

A audiometria tonal limiar foi classificada segundo Frota, $2003{ }^{11}$ :

- audição dentro faixa de normalidade: 0-20 dBNA

- perda auditiva de grau leve: 20-40 dBNA

- perda auditiva de grau moderado: 41-70 dBNA

- perda auditiva de grau severo: 71-90 dBNA

- perda auditiva de grau profundo: maior que 95 dBNA

Os limiares auditivos em campo livre com as próteses auditivas indicadas foram retirados dos prontuários e também considerados para a determinação da melhor e da pior orelha. Foi realizada audiometria em campo livre com audiômetro Madsen Midimate 622, em que os valores máximos permitidos pelo amplificador (Free Field Amplifier-F. F.) são observados na Figura 1.

Para o cálculo das médias, quando os limiares estavam ausentes foi usado valor de $130 \mathrm{~dB}$.

Foi considerado benefício com a amplificação sonora os limiares audiométricos em campo livre que permitiam acesso aos sons da fala do português falado no Brasil, segundo o estudo de Russo e Behlau (1993) ${ }^{12}$.

Para calcular a frequência de pacientes que se beneficiaram com amplificação sonora convencional, foi realizado levantamento dos pacientes atendidos no setor de prótese auditiva, que após a experiência domiciliar de 15 dias e o uso contínuo de pelo menos um mês, deram seguimento ao processo de avaliação fonoaudiológica no Grupo de Implante Coclear. Durante a avaliação, foi realizada pesquisa de limiares tonais e reconhecimento de fala e, depois de finalizados, os casos foram discutidos pela equipa multidisciplinar. Então, foram analisadas as avaliações fonoaudiólogicas no momento do estudo e foram agrupados em:

- finalizada a avaliação fonoaudiológica e considerado como candidato ao implante;

- finalizada a avaliação fonoaudiológica e dispensado pelo ganho funcional satisfatório;

- não finalizada ou não iniciada avaliação fonoaudiológica;

- dispensado pela Equipe de Implante Coclear por não preencher os critérios para IC ${ }^{5}$ ou desistência na avaliação fonoaudiológica;

- usuários de implante coclear ou implante de tronco encefálico monoaural ou candidatos ao implante de tronco encefálico.
O trabalho foi aprovado pelo Comitê de Ética e Pesquisa - CAPPesp da Diretoria do Hospital das Clínicas e da FMUSP pelo protocolo número 1039/08.

Uma vez que os limiares auditivos não apresentavam distribuição normal, foi utilizado o teste não paramétrico de Mann-Whitney para a comparação entre os grupos com ganho funcional satisfatório e insatisfatório. Foi considerado $p<0,05$ como valores estatisticamente significantes.

\section{RESULTADOS}

Dos pacientes que foram convocados para iniciar o processo de avaliação como possíveis candidatos ao implante coclear ( $\mathrm{N}=324), 164$ foram encaminhados para o setor de prótese auditiva (50\%); 11 eram pacientes do Grupo de Implante e usuários de implante coclear ou implante de tronco encefálico monoaural; 28 pacientes já estavam em processo de avaliação como candidatos ao implante coclear, sendo assim, 194 pacientes preencheram os critérios de inclusão.

Destes, 108 já eram usuários de próteses auditivas (55,6\%), 109 eram crianças (56\%) e 85 eram adultos (44\%). A média de idade foi 4,8 anos para crianças e nos adultos de 41,9 anos, sendo idade mínima 4 meses e máxima de 81 anos. 100 eram do sexo masculino (52\%) e 94 do sexo feminino (48\%).

Em relação à escolaridade, entre os adultos, 24 pacientes possuíam ensino fundamental incompleto (33\%) e 21 , ensino médio completo (29\%).

As Figuras 2 e 3 mostram o intervalo interquartil e valores míninos e máximos dos limiares de cada frequência da audiometria tonal limiar na melhor e pior orelha respectivamente nas crianças e nas Figuras 4 e 5, mostram os limiares na melhor e pior orelha respectivamente nos adultos. A média dos limiares audiométricos de 500, 1000 e $2000 \mathrm{~Hz}$ foi de 106 dBNA e 110 dBNA, melhor e na pior orelhas, respectivamente nas crianças e 118 dBNA para ambas as orelhas nos adultos.

As Figuras 6 e 7 mostram o intervalo interquartil e valores míninos e máximos dos limiares de cada frequência em campo livre com a prótese auditiva na melhor e pior orelha respectivamente nas crianças e nas Figuras 8 e 9, dos limiares na melhor e pior orelha respectivamente nos adultos. No grupo 


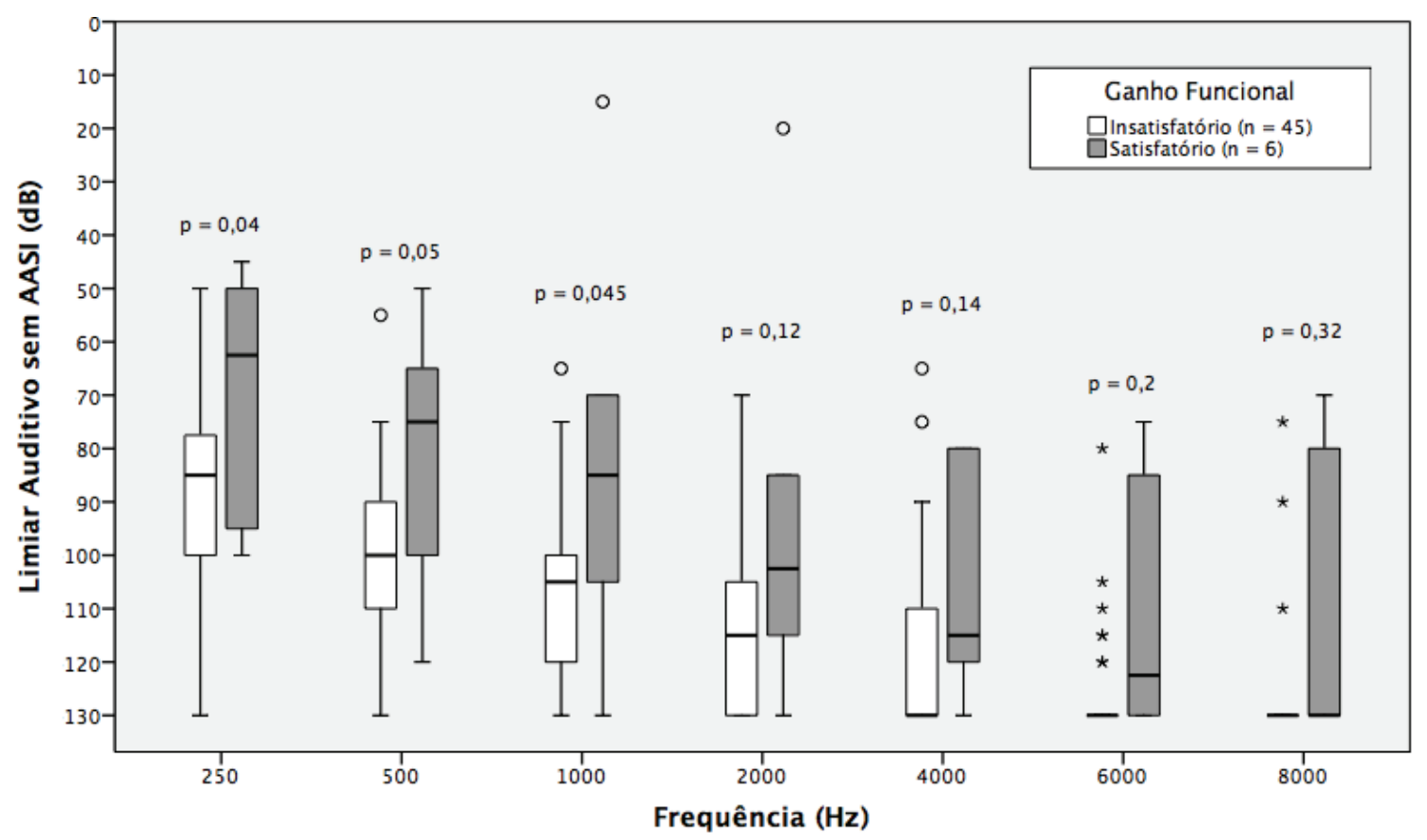

Figura 2 - Perfil audiométrico sem prótese auditiva nas crianças na melhor orelha

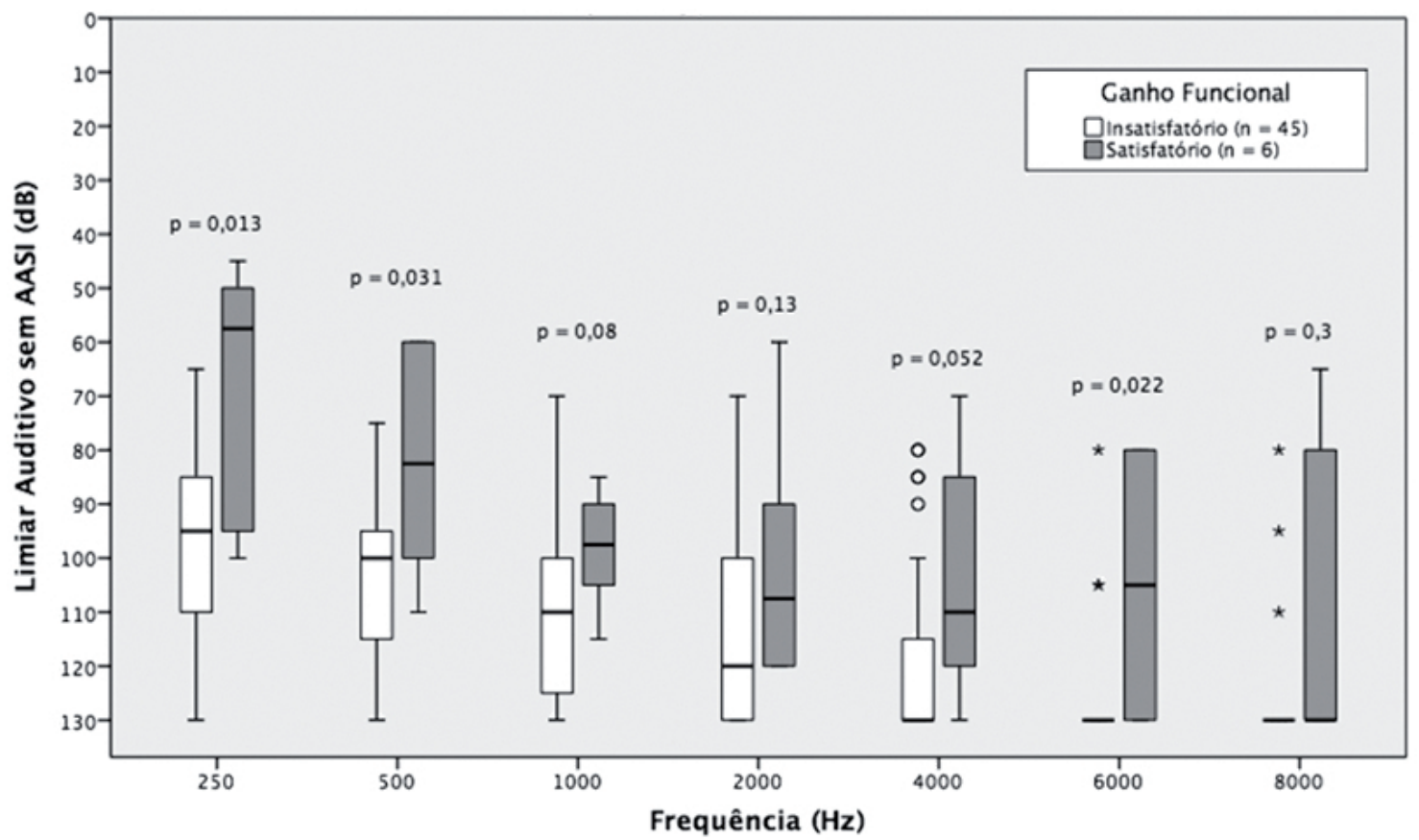

Figura 3 - Perfil audiométrico sem prótese auditiva nas crianças na pior orelha

de pacientes que não se beneficiaram da prótese auditiva, a média dos limiares de 500, 1000 e 2000 $\mathrm{Hz}$ realizado em campo livre com a prótese auditiva foi 75 dBNA na melhor orelha e 91 dBNA na pior orelha, respectivamente, nas crianças e 80 dBNA na melhor orelha e $81 \mathrm{dBNA}$ na pior orelha respectivamente nos adultos. No grupo de pacientes que se beneficiaram da prótese auditiva, a média foi $47 \mathrm{dBNA}$ na melhor orelha e $48 \mathrm{dBNA}$ na pior oreIha nas crianças e 50 dBNA na melhor orelha e 45 dBNA na pior orelha nos adultos.

A Figura 10 mostra a média de limiares em campo livre com a prótese auditiva dos 19 pacientes indicados para o uso da prótese auditiva por 


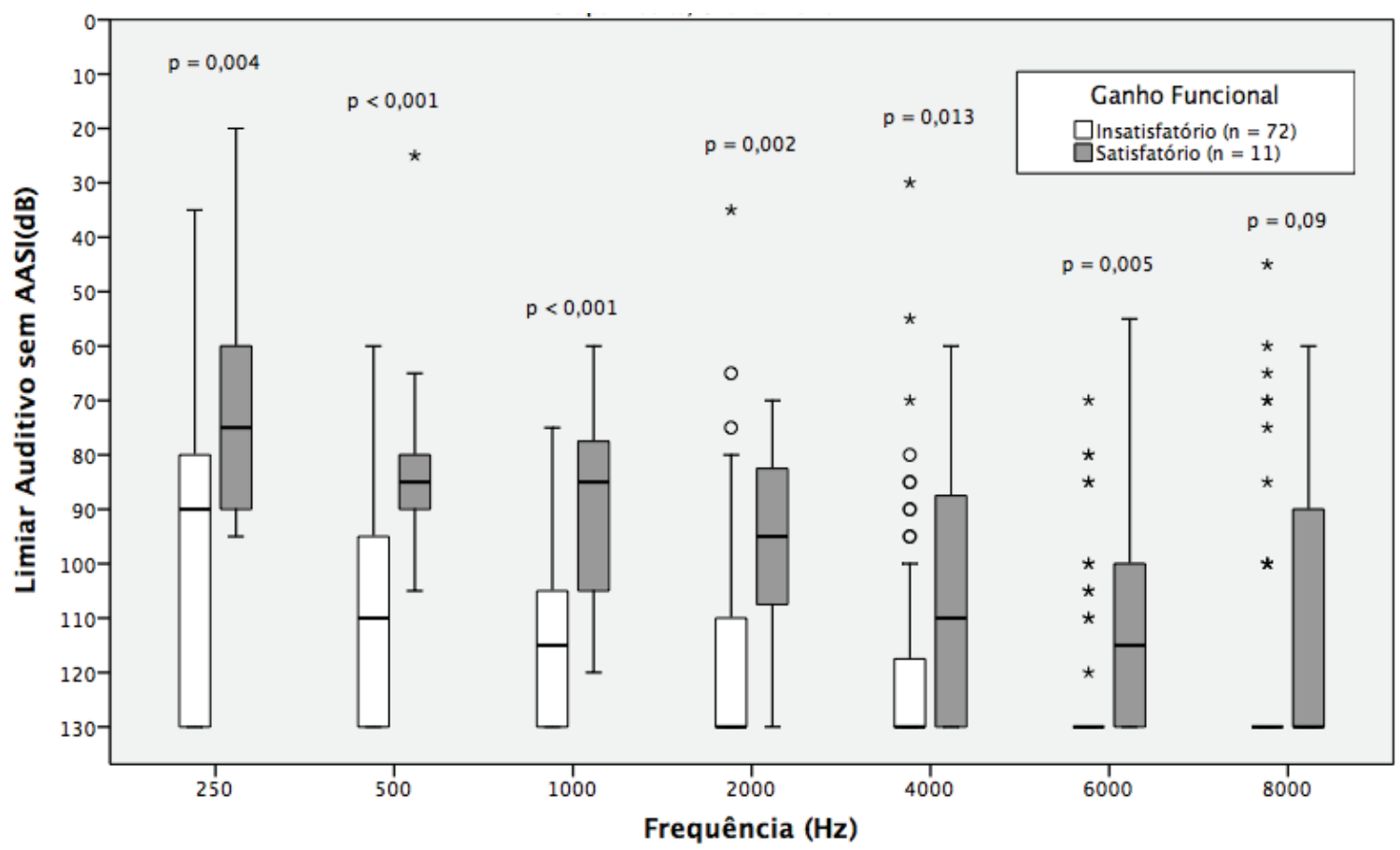

Figura 4 - Perfil audiométrico sem prótese auditiva nos adultos na melhor orelha

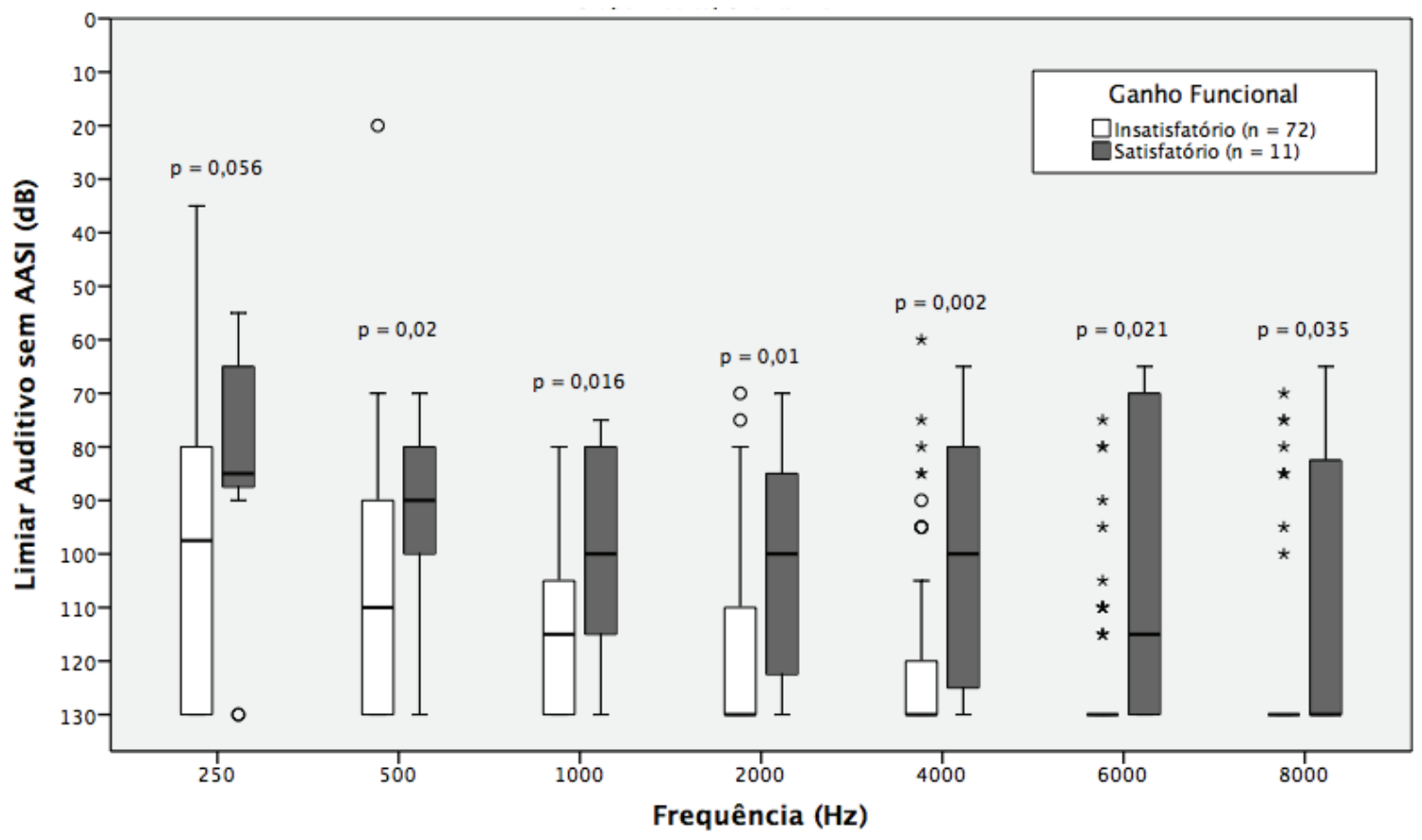

Figura 5 - Perfil audiométrico sem prótese auditiva nos adultos na pior orelha

possuírem acesso os sons da fala pelo audiograma dos sons da fala do português brasileiro ${ }^{12}$.

Em relação à etiologia, 64 eram desconhecidas (33\%), 26 eram progressivas (14\%); 24 congênitas (13\%); 21 por meningite (11\%) e 56 por outras causas $(29 \%)$.

Dos pacientes atendidos no setor de prótese auditiva, 76 (39\%) não finalizaram ou não iniciaram avaliação fonoaudiológica; 72 (37\%) finalizaram a avaliação fonoaudiológica e foram considerados candidatos ao implante; 19 (10\%) finalizaram a avaliação fonoaudiológica e foram dispensados pelo ganho funcional satisfatório, sendo 8 crianças e 11 adultos; 16 (8\%) foram dispensados pela Equipe de 
Implante Coclear por não preencherem os critérios para IC ${ }^{5}$ ou desistiram da avaliação fonoaudiológica; 11 (6\%) eram usuários de implante coclear ou implante de tronco encefálico monoaural ou candidatos ao implante de tronco encefálico.

\section{Frequência (em Hz)}

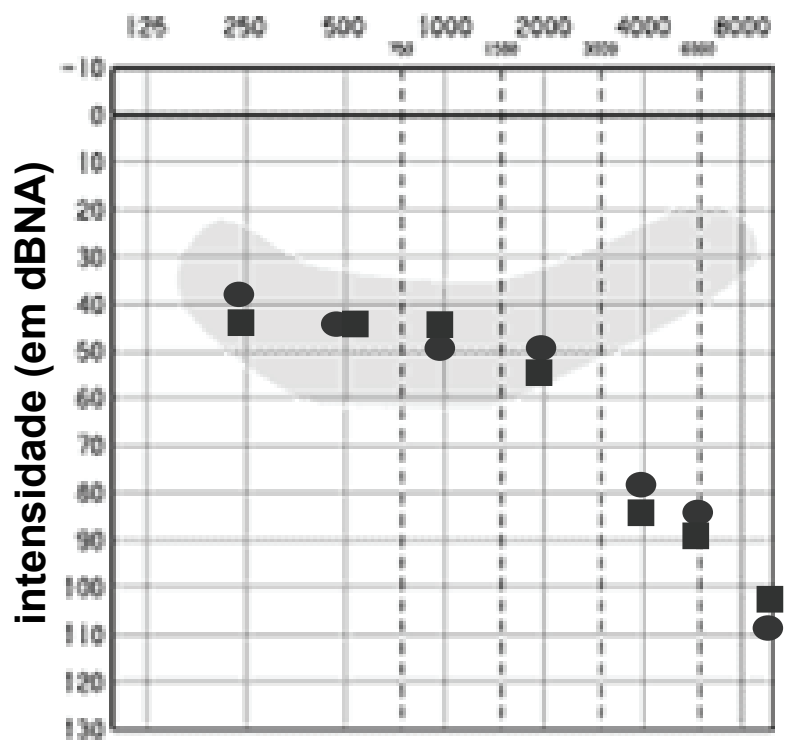

Figura 6 - Média dos limiares auditivos em campo livre com prótese auditiva nas crianças na melhor orelha

\section{DISCUSSÃO}

A média dos limiares audiológicos de todos os pacientes atendidos no setor de prótese auditiva como perda auditiva neurossensorial profunda bilateral, mostra a indicação correta destes pacientes como possíveis candidatados ao implante coclear $^{4,13}$.

Os adultos, com ganho funcional satisfatório, obtiveram os melhores limiares auditivos quando comparados ao grupo com ganho funcional insatisfatório, com diferença estatisticamente significante para todas as frequências (exceto para $250 \mathrm{~Hz}$, na pior orelha). Este foi o grupo com maior resíduo auditivo (Figuras 4 e 5). Nos limiares auditivos de ambos os grupos, não foram observadas diferenças estisticamente significantes nas crianças na maioria das frequências (Figuras 1 e 2). Porém, devese levar em consideração que o grupo de crianças com ganho funcional satisfatório foi composto de apenas seis indivíduos com resultados completos.

Não houve diferença entre o sexo na amostra. Em relação à etiologia, a de maior ocorrência foi a desconhecida ${ }^{14}$.

Apesar da maioria dos pacientes já ser usuária de próteses auditivas, o encaminhamento ao setor de prótese foi necessário, pois o uso inadequado (unilateral ou não efetivo), aparelhos com defeito, mal conservado ou inadequado para grau da perda

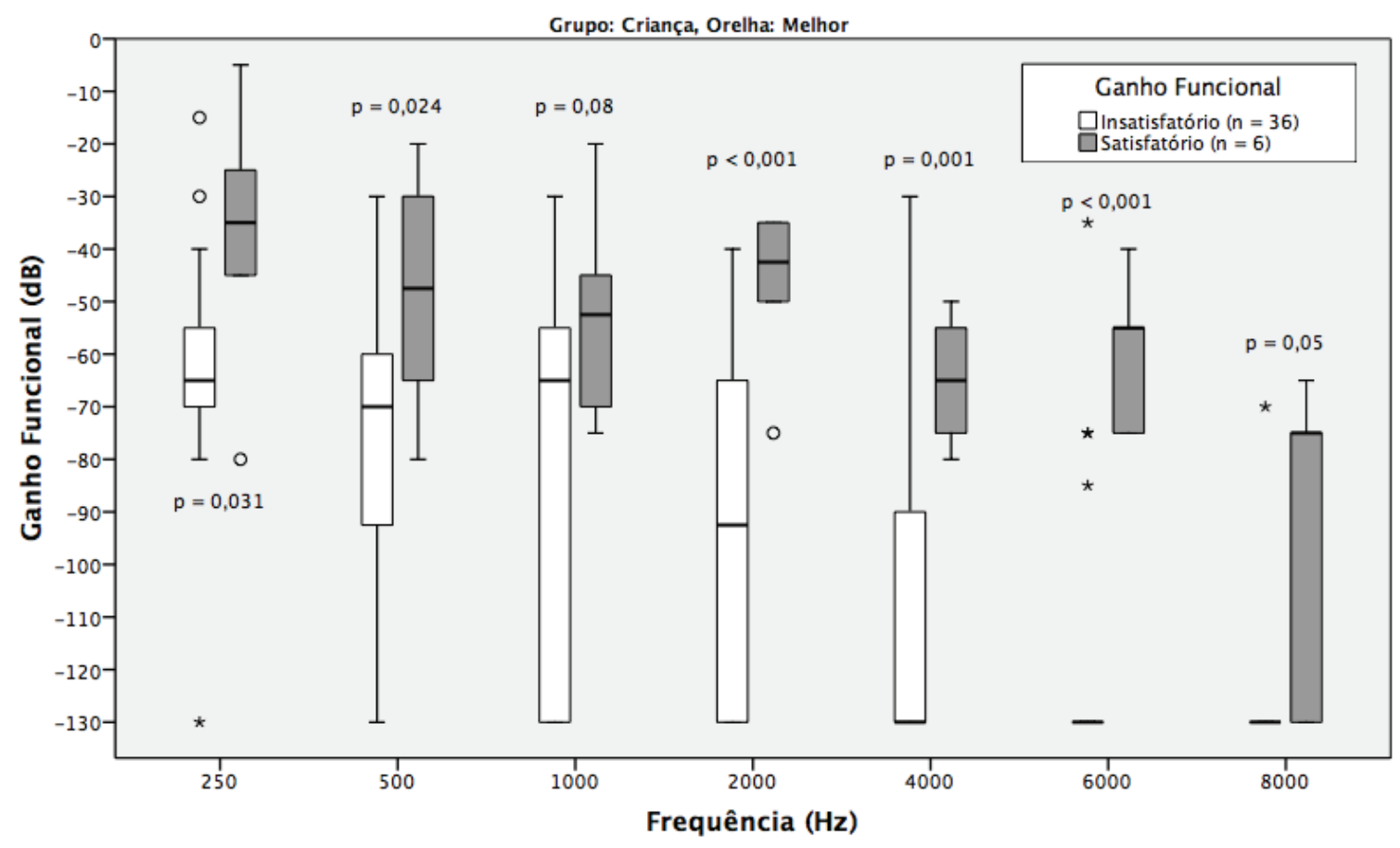

Figura 7 - Média dos limiares auditivos em campo livre com prótese auditiva nas crianças na pior orelha 


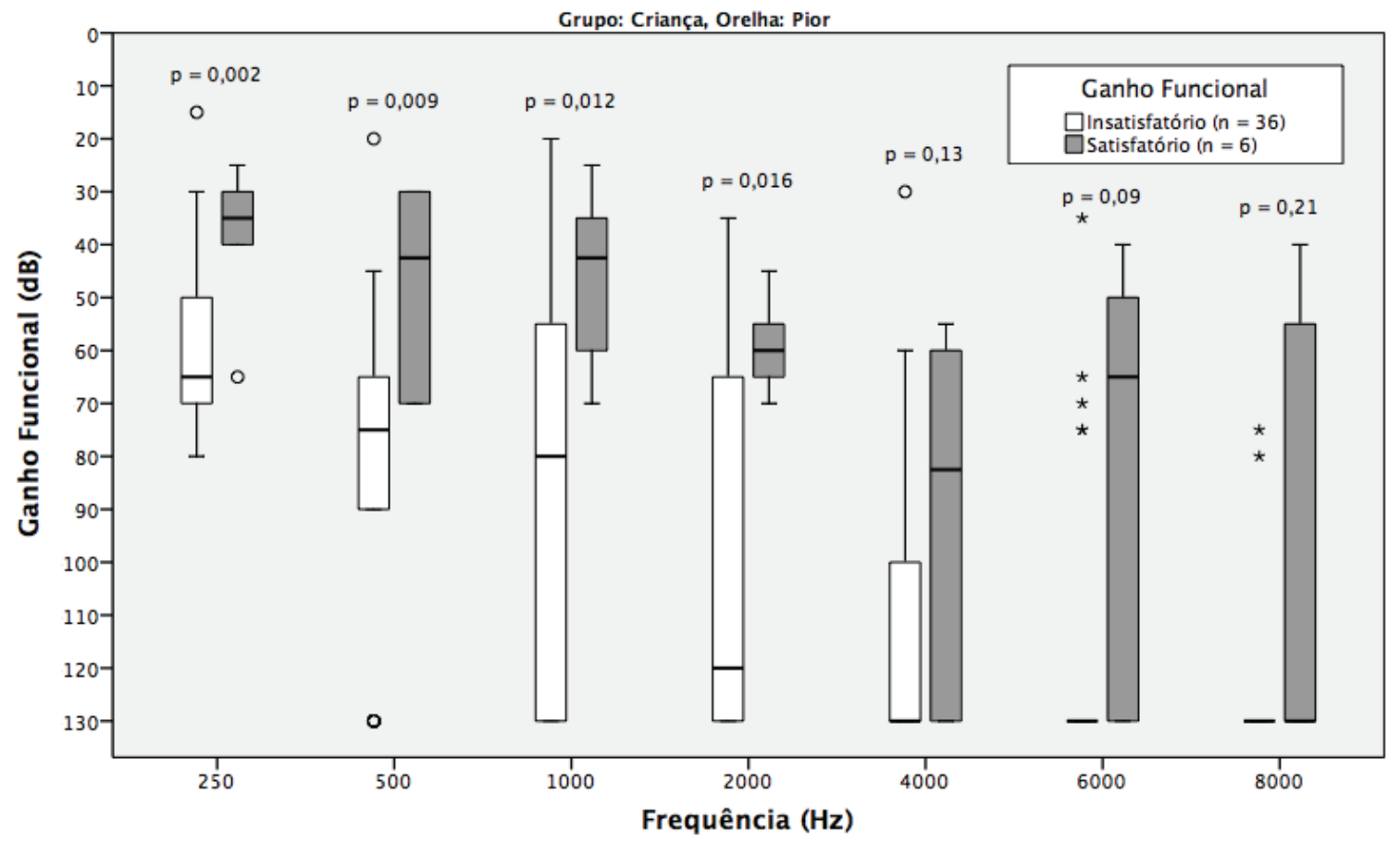

Figura 8 - Média dos limiares auditivos em campo livre com prótese auditiva nos adultos na melhor orelha

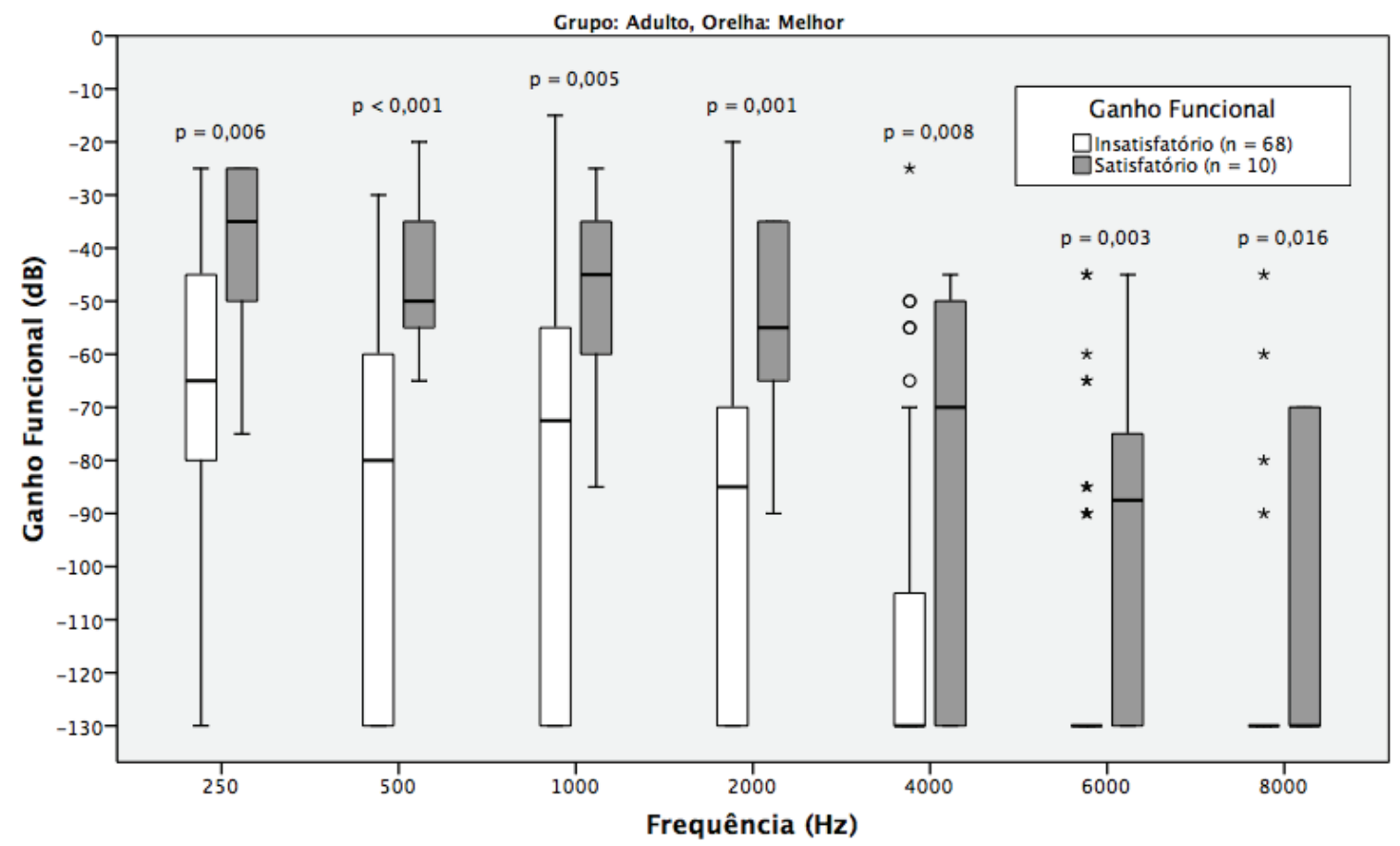

Figura 9 - Média dos limiares auditivos em campo livre com prótese auditiva nos adultos na pior orelha

auditiva, foram os motivos mais evidentes para nova adaptação e seleção.

Um dos critérios audiológicos, entre outros, para que o paciente seja considerado candidato ao implante coclear é que, apesar da experiência auditiva efetiva com próteses auditivas adequadas, não haja benefício com as mesmas ${ }^{4,13}$. A média da audiometria em campo livre dos limiares auditivos com a prótese revelou a ausência de acesso os sons da fala, o que indica audiologicamente, 


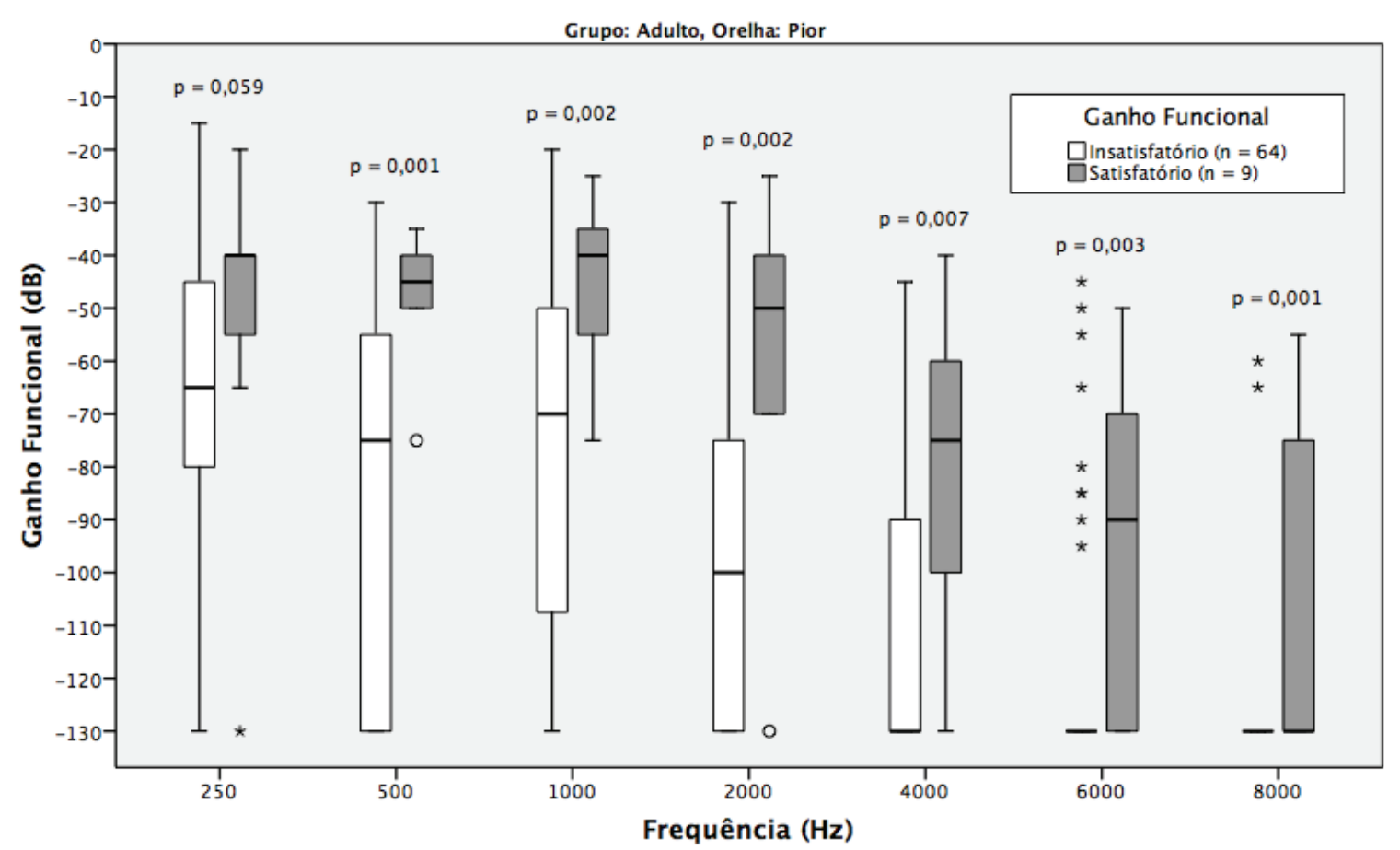

Figura 10 - Média dos limiares auditivos dos 16 pacientes com indicação do uso das próteses auditivas (adultos e crianças)

o paciente para o implante coclear ${ }^{4,5,15}$. Nesse estudo, 72 (37\%) foram considerados candidatos ao implante. Sendo que $48(24 \%)$ ainda estavam em processo de avaliação e 28 (14\%) não haviam iniciado a avaliação fonoaudiológica na ocasião do estudo.

Dos pacientes avaliados, foi indicado o uso da prótese auditiva para $19(10 \%)$, pois a média dos limiares auditivos destes pacientes mostrava acesso aos sons da fala (Figura 10).

Destes 19 pacientes, $9(47,5 \%)$ apresentaram benefícios e bons resultados com as próteses auditivas convencionais, não obtendo critérios audiológicos para indicação do implante coclear ${ }^{13}$. Porém, $10(52 \%)$ apresentaram bons limiares audiométricos, mas os testes de fala mostraram desempenho aquém ao esperado e foram encaminhados para treinamento auditivo e/ou de linguagem. Estes pacientes mantêm acompanhamento periódico para reavaliação caso haja decréssimo do desempenho ou progressão da perda de audição.

Apesar de alguns adultos e algumas crianças alcançarem limiares com a prótese auditiva satisfatórios, eles foram considerados como ganho funcional insatisfatório, pois este benefício com a prótese foi apenas em algumas frequências específicas que não eram suficientes para o reconhecimento da fala (Figuras 6,7,8 e 9).
O estudo de Teixeira et al. (2008) ${ }^{16}$ mostrou que o grau de satisfação da prótese auditiva independeu do grau da perda auditiva, observando que a perda auditiva severa a profunda pode trazer benefícios, muitas vezes associada com treinamento auditivo.

Os nove usuários de implante coclear ou implante de tronco encefálico monoaural e os dois candidatos ao implante de tronco encefálico foram atendidos no setor de prótese auditiva com a intenção de obter a binauralidade. O estudo de Ching et al., $2004{ }^{17}$ mostra os benefícios da binauralidade com a estimulação bimodal, isto é, o uso concomitante do implante com a prótese auditiva contralateral e a importância da adaptação da prótese auditiva nestes pacientes.

A adaptação da prótese auditiva contralateral ao implante torna-se necessária quando o paciente é um possível candidato ao implante bilateral. A avaliação da percepção de fala e dos limiareas auditivos com a prótese audtiva, bem como os benefícios da estimulação bimodal serão dados importantes para decisão do implante bilateral ${ }^{17}$.

A etapa de adaptação e seleção de próteses auditivas é fundamental para dar continuidade ao processo de avaliação fonoaudiológica para o implante coclear. O ambulatório de prótese auditiva da equipe do HCFMUSP tem como principal objetivo facilitar este processo, tornando mais eficiente 
e rápida a adaptação e seleção dos candidatos à cirurgia.

Uma vez que diversos estudos mostraram que há maior benefício com o implante coclear em crianças implantadas até os dois anos de idade, que é o período idade ideal para melhor aproveitamento dos resultados do implante coclear ${ }^{18,19}$, mostrando que a criança pode desenvolver-se próxima da normalidade ${ }^{20}$.

A maioria dos casos foi considerada como apta ao implante coclear, o que reflete o objetivo deste ambulatório. Porém, a presença de 19 pacientes indicados ao uso das próteses auditivas convencionais mostra a importância da adaptação se seleção mesmo nos casos de perda auditiva severa a profunda bilateralmente.

\section{CONCLUSÃO}

O perfil dos pacientes candidatos ao implante coclear os quais passaram no setor de próteses auditivas é:

- na maioria, criança, do sexo masculino;

- de etiologia desconhecida;

- já usuário de próteses auditivas;

- média de idade de 4,8 anos para crianças e 41,9 anos nos adultos;

- audiometria com perda auditiva neurossensorial profunda bilateral;

- 10\% (19) dos pacientes encaminhados como candidatos ao IC puderam se beneficiar da amplificação da prótese auditiva convencional.

\begin{abstract}
Purpose: to characterize the audiometric and demographic profile of cochlear implant candidates who were referred to hearing aid sector, and if they benefit from the amplification of the hearing aid. Methods: patients who had been attended from May 2007 to December 2008 were studied. We collected the patients' profile involving: gender, age, education and etiology. The hearing threshold's average for the good and worse ears was calculated and classified according to Frota (2003). The averaged sound field thresholds with hearing aid was calculated for both ears and considered as a benefit when allowed for an access to the speech sounds of Brazilian Portuguese. Results: a hundred and ninety-four medical records were evaluated in this period. Among them, 108 patients had already been wearing hearing aids (55.6\%), 100 were male (52\%), 94 female (48\%), 109 were children (56\%) and 85 adults (44\%). The average age was 4.8year old for children and 41.9-year old for adults. Among the adults, 24 patients had incomplete high school education (33\%). Unknown etiology was the most frequent cause (33\%). Nineteen patients have benefited from the use of hearing aids (10\%). The averaged sound field thresholds with hearing aid was $47 \mathrm{dBHL}$ and $48 \mathrm{dBHL}$, in best and worse ears, respectively, for children, and $50 \mathrm{dBHL}$ and $45 \mathrm{dBHL}$, respectively, for adults. Conclusions: the profile of the patients was: majority of children, males, unknown etiology, previously hearing aid users, average of age 4.8-year old for children and 41.9 for adults. Pure tone thresholds revealed profound bilateral hearing loss. About $10 \%$ of patients were benefited with the use of hearing aids.
\end{abstract}

KEYWORDS: Cochlear Implantation; Hearing; Hearing Aids; Hearing Loss; Speech

\section{REFERÊNCIAS}

1. Pereira MB, Feres MCLC. Próteses auditivas. Medicina. 2005; 38(3/4):257-61.

2. Bevilacqua MC. A ética em programas de implante coclear em crianças. Bol. Psicol. 2001; 51(115):169-76.

3. Oliveira JAA. Implante coclear. Rev. Medicina. 2005; 38(3/4):262-72.

4. Bento RF, Brito Neto R, Castilho AM, Gómez MVSG, Giorgi SB, Guedes MC. Resultados auditivos com o implante coclear multicanal em pacientes submetidos a cirurgia no Hospital das Clínicas da Faculdade de Medicina da Universidade de São Paulo. Rev. Bras. Otorrinolaringol. 2004; 70(5):632-7.

5. Goffi-Gomez MVSG, Guedes MC, Sant'Anna SBG, Peralta CGO, Tsuji RK, Castilho AM, et al. Critérios de seleção e avaliação médica e audiológica dos candidatos ao implante coclear: protocolo HC-FMUSP. Arq. Otorrinolaringol. 2004; 8(4):303-23.

6. Gordon KA, Twitchell KA, Papsin BC, Harrison $R V$. Effect of residual hearing prior to cochlear 
implantation on speech perception in children. $\mathrm{J}$ Otolaryngol. 2001; 30(4):216-23.

7. Banhara MR, Nascimento LT, Costa Filho AO, Bevilacqua MC. Uso combinado do implante coclear e aparelho de amplificação sonora individual em adultos. Dist Comun. 2004; 16(1):27-33.

8. Morera C, Manrique M, Ramos A, Garcia-lbanez L, Cavalle L, Huarte A, et al. Advantages of binaural hearing provided through bimodal stimulation via a cochlear implant and a conventional hearing aids: a 6-month comparative study. Acta Otolaryngol. 2005; 125(6):596-606. http://dx.doi. org/10.1080/00016480510027493

9. Ching TY, Hill M, Brew J, Incerti P, Priolo S, Rushbrook E, et al. The effect of auditory experience on speech perception, localization, and functional performance of children who use a cochlear implant and a hearing aid in opposite ears. Int $\mathrm{J}$ Audiol. 2005; 44(12):677-90.

10. Ching TYC, Incerti P, Hill M, Van Warrooy E. An overview of binaural advantages for children and adults who use binaural/bimodal hearing devices. Audiol Neurotol. 2006; 11(suppl1):6-11. http:// dx.doi.org/10.1159/000095607

11. Frota S. Avaliação básica da audição. In: Frota $\mathrm{S}$, organizador. Fundamentos em fonoaudiologia: audiologia. 2. ed. Rio de Janeiro: Guanabara Koogan; 2003. p. 41-60.

12. Russo ICP, Behlau M. As pistas acústicas das vogais e consoantes. In: Russo ICP, Behlau M, organizadores. Percepção da fala: análise acústica do Português brasileiro. São Paulo: Lovise; 1993. p. 25-50.
13. Protocolo Latino Americano para Implantes Cocleares. Elaborado pelo Grupo de Pesquisas Latino-americano. Cochlear Américas; 2003.

14. Pupo AC, Balieiro CR, Figueiredo RSL. Estudo retrospectivo de crianças e jovens com deficiência auditiva: caracterização das etiologias e quadro audiológico. Rev. CEFAC. 2008; 10(1):84-91. http:// dx.doi.org/10.1590/S1516-18462008000100012

15. Moraes TV, Zeigelboim BS, Bevilacqua MC, Jacob LCB. Indicaçäo de implante coclear: tendências atuais. Acta AWHO. 2001; 20(4):229-37. 16. Teixeira CF, Augusto LGS, Caldas Neto SS. Prótese auditiva: satisfação do usuário com sua prótese e com seu meio ambiente. Rev. CEFAC. 2008; 10(2):245-53. http://dx.doi.org/10.1590/ S1516-18462008000200015

17. Ching TYC, Incerti $P$, Hill M. Binaural benefits for adults who use hearing aids and cochlear implants in opposite ears. Ear Hear. 2004; 25(1):9-21.

18. Miyamoto RT, Hay-McCutcheon MJ, Kirk KI, Houston DM, Bergeson-Dana T. Language skills of profoundly deaf children who received cochlear implants under 12 months of age: a preliminary study. Acta Otolaryngol. 2008; 128(4):373-7. http:// dx.doi.org/10.1080/00016480701785012

19. Profant M, Kabatova Z, Simkova L. From hearing screening to cochlear implantation: cochlear implants in children under 3 years of age. Acta Otolaryngol. 2008; 128(4):369-72. http://dx.doi. org/10.1080/00016480701736254

20. Stuchi RF, Nascimento LT, Belivacqua MC, Brito Neto RV. Linguagem oral de crianças com cinco anos de uso do implante coclear. Pró-Fono. 2007; 19(2):167-76.

DOI: 10.1590/S1516-18462010005000033

RECEBIDO EM: 30/08/2009

ACEITO EM: 15/12/2009

Endereço para correspondência:

Ana Tereza de Matos Magalhães

Rua Capote Valente, 432 conj. 14

São Paulo - SP

CEP: 05409-001

E-mail: atmmagalhaes@yahoo.com.br 